\title{
Perfil dos concluintes de Odontologia da Universidade Estadual da Paraíba
}

\author{
Jeanne Paula Ferreira de Oliveira Querino*; Larissa Rangel Peixoto**; Gêisa Aiane de Morais Sampaio** \\ * Discente do curso de Graduação em Odontologia da \\ Universidade Estadual da Paraíba, Campus Araruna \\ ** Docente do Curso de Odontologia da Universidade Estadual \\ da Paraíba, Campus Araruna
}

Recebido em 14/02/2017. Aprovado em 18/05/2017.

\begin{abstract}
RESUMO
O estudo teve como objetivo identificar o perfil sociodemográfico dos estudantes do último ano de Odontologia da Universidade Estadual da Paraíba (UEPB) em 2016, os motivos que os levaram a optar pela Odontologia, a satisfação com o curso, bem como suas perspectivas de trabalho e de pósgraduação. A amostra foi constituída por 60 estudantes, que responderam a um questionário semiestruturado dividido em três blocos: perfil sociodemográfico; curso de graduação em Odontologia; e perspectivas de atuação profissional e de pós-graduação. Os resultados mostraram um predomínio de mulheres, jovens, solteiras, sem filhos, naturais do estado da Paraíba, com renda familiar mensal de 3 a 5 salários mínimos e sem familiares atuando como cirurgiões-dentistas. Seus pais apresentavam ensino médio ou superior completos e estavam inseridos no mercado de trabalho. Mais de $90 \%$ mostrou-se satisfeito com a opção pela Odontologia. Os motivos que os levaram a optar pela profissão foram a realização pessoal e profissional, seguida pelo interesse em atuar na comunidade. Cerca de $40 \%$ dos estudantes referiram reprovação e poucos referiram trancamento de matrícula no curso por algum período. Cerca de $80 \%$ dos estudantes avaliaram o curso como bom ou ótimo e consideram adequado o tempo de graduação de cinco anos. Pretendem trabalhar tanto no serviço público quanto no privado e fazer pós-graduação. Os achados permitiram identificar o perfil do formando em Odontologia da UEPB em 2016. Recomenda-se o acompanhamento permanente dos formandos como um importante instrumento de monitoramento de seu perfil e do desenvolvimento do currículo.

Descritores: Odontologia. Educação Superior. Estudantes de Odontologia.
\end{abstract}

\section{INTRODUÇÃO}

A odontologia brasileira tem apresentado mudanças tanto no setor de novas tecnologias quanto no rumo que o profissional deve tomar diante das dificuldades do mercado de trabalho ${ }^{1}$. A inserção dos profissionais de saúde bucal nos diversos níveis de atenção à saúde e na gestão de serviços de saúde no Brasil reforça a necessidade de reflexão sobre a prática profissional com responsabilidade e ética na produção do cuidado $^{2}$.

O papel da universidade, especialmente dentro da Área da Saúde, é desenvolver competências e formar um profissional capaz de atender as demandas da população, buscando a relevância social do ato profissional ${ }^{2}$, eliminando, 
assim, a tendência de formação predominantemente elitista, com pouca preocupação com o social e a promoção de saúde, que se observava anteriormente $^{3}$. As Diretrizes Curriculares Nacionais (DCN) em Odontologia dispõem que o novo profissional deve ter uma formação generalista, crítica, humanista e reflexiva, que lhe permita trabalhar em diferentes níveis de atenção do sistema de saúde 4 . O curso de Odontologia deve, portanto, objetivar a formação de recursos humanos voltados para o perfil epidemiológico da população como também para o sistema de saúde vigente no Brasil $^{2}$.

As transformações no cenário acadêmico brasileiro ocorridas nas últimas décadas, causaram mudanças no perfil do estudante de educação superior no Brasil. Estas transformações foram decorrentes, principalmente, da expansão de vagas e criação de novas instituições públicas e das políticas de inserção de estudantes pertencentes a grupos sociais tradicionalmente desfavorecidos ${ }^{5}$. Os Programas de Ações Afirmativas, por exemplo, visam combater a desigualdade em relação às oportunidades educacionais, com o propósito de potencializar o acesso de grupos minoritários à educação superior ${ }^{6}$.

Estudos têm evidenciado o perfil e as expectativas de estudantes de Odontologia em relação à profissãa ${ }^{1,7-10}$. As motivações que levam esses estudantes à escolha do curso têm relação com a visão que os mesmos apresentam da Odontologia, das possibilidades de atuação no mercado de trabalho e da necessidade ou não de comprometimento social enquanto profissionais de saúde ${ }^{7}$. A vocação ou realização profissional tem demonstrado ser o principal motivo de escolha da profissão por estudantes de odontologia $^{1,8,9,11}$, bem como as razões econômicas, por a considerarem uma profissão que oferece estabilidade financeira ${ }^{12}$.

A visão dos graduandos sobre o mercado de trabalho mostra que grande parte destes acredita que há espaço para profissionais especializados ${ }^{11}$. A exigência do mercado de um profissional em constante aperfeiçoamento explica o anseio em fazer cursos de pósgraduação ${ }^{1,8-10}$. Observa-se também alta pretensão de associação das práticas nos setores público e privado, provavelmente devido a fatores relacionados aos serviços públicos brasileiros, que geralmente pagam baixos salários, mas oferecem segurança no emprego e também podem permitir que os trabalhadores se dediquem à prática privada ${ }^{10}$.

A Universidade Estadual da Paraíba (UEPB) oferece atualmente dois cursos de Odontologia, o primeiro localizado na cidade de Campina Grande/PB (Campus I), criado em 1971, que possui carga horária de 4505 horas e oferta 30 vagas de ingressos por semestre. O segundo, situado na cidade de Araruna/PB (Campus VIII), foi criado em 2010, possui carga horária de 4220 horas e 40 vagas ofertadas por semestre. Ambos requerem tempo mínimo de formação de 10 e máximo de 15 semestres. Os mesmos possuem políticas de atividades de monitoria e projetos de pesquisa e extensão ${ }^{13,14}$.

Para aproximar as atividades de ensino, pesquisa e extensão e melhor articulá-las, o Regimento dos Cursos de Graduação da UEPB abre a possibilidade de que as atividades desenvolvidas em projetos de pesquisa de extensão sejam integralizadas pelos estudantes como carga horária de estágio supervisionado ou como atividade complementar de natureza científico-acadêmico-cultural ${ }^{13,14}$. Atualmente, o Departamento de Odontologia Campus I desenvolve 23 projetos e 6 programas de extensão e apresenta 6 linhas de pesquisa; ${ }^{13}$ o departamento de Odontologia Campus VIII apresenta 3 linhas de extensão e 3 linhas de pesquisa $^{14}$.

A UEPB oferta $100 \%$ de suas vagas de 
nível superior por meio do Sistema de Seleção Unificada (SiSU) do Ministério da Educação (MEC), que utiliza a pontuação do candidato obtida no Exame Nacional do Ensino Médio (ENEM) como processo de avaliação ${ }^{13,14}$.

Grande parte dos estudos sobre formação do cirurgião-dentista no Brasil revelam a necessidade de mudanças na formação do cirurgião-dentista, nos currículos, no ensino voltado para as necessidades sociais e maior integração ensino-serviço ${ }^{3,7}$. Portanto, exige-se aprofundamento nesse campo para melhor compreensão das questões relacionadas ao perfil dos acadêmicos de Odontologia, suas motivações e expectativas profissionais.

$\mathrm{O}$ presente estudo propõe-se a analisar o perfil sociodemográfico dos estudantes de Odontologia da UEPB regularmente matriculados no último ano do curso, os motivos que os levaram a escolher a Odontologia, apreciação do curso, bem como as perspectivas de emprego e pós-graduação.

\section{METODOLOGIA}

Trata-se de um estudo transversal observacional descritivo. O universo do estudo correspondeu a todos os alunos regularmente matriculados no último ou penúltimo semestre dos cursos de Odontologia da UEPB ( $n=99$ ), com previsão de formatura em 2016.1 ou 2016.2. Foram incluídos aqueles que estavam presentes no momento da aplicação do questionário e que aceitaram participar do estudo por meio da assinatura do Termo de Consentimento Livre e Esclarecido (TCLE).

Para a coleta de dados foi utilizado um questionário semiestruturado desenvolvido por Bockmann et al. (2014) ${ }^{7}$ e adequado aos objetivos do presente estudo. $\mathrm{O}$ mesmo foi dividido em 3 blocos: perfil sociodemográfico dos estudantes, curso de graduação em Odontologia, e perspectivas de atuação profissional e de pós-graduação. A aplicação do questionário ocorreu em salas de aula da UEPB e os estudantes levaram cerca de 35 minutos para respondê-lo.

A análise das informações coletadas foi realizada no programa estatístico Statistical Package for the Social Sciences (SPSS) para Windows versão 17.0. Foram calculadas as distribuições de frequência das variáveis investigadas. Uma análise descritiva dos dados foi realizada e os resultados foram agrupados em tabelas e gráficos.

A condução do estudo obedeceu aos princípios regidos pelas normas nacionais e internacionais que regulam as pesquisas com seres humanos. A pesquisa foi aprovada, pelo Comitê de Ética em Pesquisa da UEPB (CAAE $\mathrm{n}^{\mathrm{o}}$ 56416916.5.0000.5187).

\section{RESULTADOS}

Dos 99 estudantes matriculados nos últimos semestres dos cursos de Odontologia da UEPB, 16 foram excluídos por não estarem presentes durante a aplicação do questionário e 23 se recusaram a participar. Assim, foram incluídos na pesquisa 60 estudantes mediante a assinatura do TCLE, com taxa de resposta de $59,4 \%$. Destes, a maioria eram mulheres $(69,81 \%)$, jovens $(75,17 \%$ com idade entre $21 \mathrm{e}$ 25 anos), solteiros (93,33\%), sem filhos (93,33\%), naturais da Paraíba $(90,00 \%)$, com renda familiar mensal de 3 a 5 salários mínimos $(41,66 \%)$ e sem familiares atuando como cirurgiões-dentistas $(75,00 \%)$ (tabela 1$)$.

Com relação às variáveis familiares, a maioria dos pais se encontravam casados $(75,00 \%)$, possuíam ensino médio ou superior (36,67\% dos pais com ensino médio completo e $36,67 \%$ das mães com ensino superior completo) e estavam trabalhando no momento da aplicação dos questionários $(68,33 \%$ dos pais e $55,00 \%$ das mães). Para $88,34 \%$ dos estudantes o maior responsável pela renda familiar foram os pais (tabela 1). 
Tabela 1. Distribuição dos formandos do curso de Odontologia segundo a caracterização sociodemográfica

\begin{tabular}{|c|c|c|c|}
\hline Variáveis & & $\mathbf{n}$ & $\%$ \\
\hline \multirow[t]{2}{*}{ Sexo } & Feminino & 37 & 69,81 \\
\hline & Masculino & 23 & 30,19 \\
\hline \multirow[t]{2}{*}{ Idade (anos) } & 21 a 25 & 45 & 75,17 \\
\hline & 26 a 29 & 15 & 24,83 \\
\hline \multirow[t]{2}{*}{ Estado civil } & Solteiro & 56 & 93,33 \\
\hline & Casado & 4 & 6,67 \\
\hline \multirow[t]{2}{*}{ Possui filhos? } & Sim & 4 & 6,67 \\
\hline & Não & 56 & 93,33 \\
\hline \multirow[t]{2}{*}{ Estado de origem } & Paraíba & 54 & 90,00 \\
\hline & Outro & 6 & 10,00 \\
\hline \multirow[t]{4}{*}{ Renda familiar $(S M) *$} & $1 \mathrm{a} 2 \mathrm{sm}$ & 15 & 25,00 \\
\hline & 3 a $5 \mathrm{sm}$ & 25 & 41,66 \\
\hline & Mais de $5 \mathrm{sm}$ & 7 & 11,67 \\
\hline & Não informou & 13 & 21,67 \\
\hline \multirow[t]{2}{*}{ Familiar exerce a Odontologia? } & Sim, pais ou irmãos & 7 & 25,00 \\
\hline & Não & 45 & 75,00 \\
\hline \multirow[t]{4}{*}{ Estado civil dos pais } & Casado & 45 & 75,00 \\
\hline & Divorciado & 9 & 15,00 \\
\hline & Solteiro & 3 & 5,00 \\
\hline & Viúvo & 3 & 5,00 \\
\hline \multirow[t]{6}{*}{ Escolaridade do pai } & Fundamental incompleto & 11 & 18,33 \\
\hline & Fundamental completo & 8 & 13,33 \\
\hline & Médio completo & 22 & 36,67 \\
\hline & Superior completo & 16 & 26,67 \\
\hline & Analfabeto & 1 & 1,67 \\
\hline & Não sabe informar & 2 & 3,33 \\
\hline \multirow[t]{5}{*}{ Escolaridade da mãe } & Fundamental incompleto & 9 & 15,00 \\
\hline & Fundamental completo & 9 & 15,00 \\
\hline & Médio completo & 19 & 31,66 \\
\hline & Superior completo & 22 & 36,67 \\
\hline & Analfabeto & 1 & 1,67 \\
\hline \multirow[t]{3}{*}{ Mercado de trabalho: pai } & Está trabalhando & 41 & 68,33 \\
\hline & Está desempregado & 5 & 8,33 \\
\hline & Outro & 14 & 23,34 \\
\hline \multirow[t]{3}{*}{ Mercado de trabalho: mãe } & Está trabalhando & 33 & 55,00 \\
\hline & Está desempregada & 15 & 25,00 \\
\hline & Outro & 12 & 20,00 \\
\hline \multirow[t]{3}{*}{ Responsável pela renda familiar } & Pai ou mãe & 53 & 88,34 \\
\hline & Esposo ou esposa & 3 & 5,00 \\
\hline & Outro & 4 & 6,66 \\
\hline & TOTAL & 60 & 100,00 \\
\hline
\end{tabular}

SM: salários mínimos. *Valor do salário mínimo no momento da aplicação do questionário: R\$ 880,00. 
Grande parte dos estudantes foi aprovada no primeiro vestibular $(58,33 \%)$ e $68,23 \%$ ingressou no curso com idade entre 17 e 20 anos. A Odontologia na UEPB foi o primeiro curso de graduação para $58,33 \%$ dos estudantes e $96,43 \%$ deles mostraramse satisfeitos com a opção (tabela 2).

Tabela 2. Distribuição dos formandos em relação a dados do curso de Odontologia

\begin{tabular}{|c|c|c|}
\hline Variáveis & $\mathbf{n}$ & $\%$ \\
\hline \multicolumn{3}{|l|}{ Número de vestibulares realizados } \\
\hline Aprovação no primeiro vestibular & 35 & 58,33 \\
\hline Dois ou mais vestibulares & 22 & 36,67 \\
\hline Não informou & 3 & 5,00 \\
\hline \multicolumn{3}{|l|}{ Idade de ingresso no curso (anos) } \\
\hline Entre 17 e 20 anos & 41 & 68,23 \\
\hline Mais de 20 anos & 19 & 31,77 \\
\hline \multicolumn{3}{|l|}{ Está no primeiro curso de graduação } \\
\hline Sim & 35 & 58,33 \\
\hline Não & 25 & 41,67 \\
\hline \multicolumn{3}{|l|}{ Satisfação com o curso } \\
\hline Satisfeito & 58 & 96,43 \\
\hline Pouco satisfeito & 2 & 3,77 \\
\hline TOTAL & 60 & 100,00 \\
\hline
\end{tabular}

Os principais motivos que os levaram a optar pela Odontologia foram a realização pessoal e profissional (43), seguida pelo interesse em atuar na comunidade (12), segurança e tranquilidade no futuro (10), ou influência de cirurgiões-dentistas parentes e amigos (7). Para estes estudantes, a principal finalidade da Odontologia é a prevenção e a manutenção da saúde bucal (43), assim como promover higiene e estética (40) ou a prevenção e tratamento (39).

Vários estudantes referiram pelo menos uma reprovação ao longo do curso $(43,33 \%)$ e apenas $3,33 \%$ afirmaram ter realizado trancamento de matrícula por algum período letivo, por gestação $(1,67 \%)$ ou por outros motivos pessoais $(1,67 \%)$. Cerca de $94,50 \%$ dos estudantes afirmou ter realizado monitorias, projetos de extensão e/ou pesquisa durante a graduação e 70,45\% receberam remuneração pela atividade. Os estudantes avaliaram o curso de graduação de Odontologia da UEPB como bom $(48,33 \%)$, ótimo $(31,67 \%)$, regular $(18,33 \%)$ ou ruim $(1,67 \%)$. O tempo de graduação (cinco anos) foi considerado adequado por $85,00 \%$ dos estudantes.

Depois de formados, mais da metade dos estudantes $(68,33 \%)$ pretende atuar tanto no serviço público quanto no privado. Destes, quando questionados se dedicariam 40 horas semanais para trabalhar junto a uma Equipe de Saúde Bucal na Estratégia Saúde da Família, 97,50\% afirmaram que sim (tabela 3 ).

Os motivos que levariam os estudantes a trabalhar no setor privado foram a autonomia profissional (34) e melhores condições de trabalho (29). Em relação à opção de trabalho nos setores público e privado, os motivos apontados pelos estudantes foram a renda segura, 
experiência nos primeiros anos de profissão até montar um consultório ou formar clientela (48) e maior experiência profissional (35). Com o término da graduação, a expectativa de retorno financeiro para a maior parte dos estudantes (41,67\%) foi de 6 a 10 salários mínimos (de R\$ 5.280,00 a $\mathrm{R} \$ 8.800,00)$.

Cerca de $96,67 \%$ pretende fazer cursos de pós-graduação, de modo especial cursos de especialização $(66,67 \%)$, em um período de até 2 anos $(78,33 \%)$ após a conclusão da graduação (tabela 3).

Parte dos estudantes demostrou interesse em se especializar em mais de uma área da Odontologia $58,33 \%$, enquanto que $41,67 \%$ apontaram apenas uma área de interesse. Entre as áreas mais citadas estão Prótese (24), Implantodontia (24), Endodontia (18) e Ortodontia (18).

Tabela 3. Distribuição dos formandos quanto à pretensão de vínculo de trabalho e pós-graduação

\begin{tabular}{|c|c|c|c|}
\hline Variáveis & & $\mathbf{n}$ & $\%$ \\
\hline \multicolumn{4}{|c|}{ Pretensão de vínculo de trabalho } \\
\hline & Somente em serviço público & 1 & 1,67 \\
\hline & Somente no serviço privado & 1 & 1,67 \\
\hline & Público e privado & 41 & 68,33 \\
\hline & Na universidade (docência) & 15 & 25,00 \\
\hline & Não sabe informar & 2 & 3,33 \\
\hline \multicolumn{4}{|c|}{ Atuação na estratégia de saúde da família (40 horas) } \\
\hline & Pretende & 40 & 97,50 \\
\hline & Não pretende & 1 & 2,50 \\
\hline \multicolumn{4}{|c|}{ Pós-graduação } \\
\hline & Sim & 58 & 96,67 \\
\hline & Não & 2 & 3,33 \\
\hline \multicolumn{4}{|c|}{ Até onde pretende continuar se aperfeiçoando } \\
\hline & Pós-graduação (especialização) & 40 & 66,67 \\
\hline & Pós-graduação (mestrado e doutorado) & 16 & 26,67 \\
\hline & Não pretende se aperfeiçoar & 2 & 3,33 \\
\hline & Não sabe informar & 2 & 3,33 \\
\hline \multicolumn{4}{|c|}{ Período de tempo para pós-graduação } \\
\hline & Até 1 ano depois da graduação & 45 & 75,00 \\
\hline & Até 2 anos depois da graduação & 2 & 3,33 \\
\hline & Mais de 2 anos depois da graduação & 10 & 16,67 \\
\hline & Não sabe informar & 7 & 11,67 \\
\hline$\overline{\text { TOTAL }}$ & & 60 & $\mathbf{1 0 0 , 0 0}$ \\
\hline
\end{tabular}




\section{DISCUSSÃO}

Os estudantes participantes da pesquisa eram, em sua maioria, mulheres, jovens, solteiras, sem filhos e do mesmo estado de origem que a instituição. Essas mesmas características foram identificadas em outros estudos sobre o perfil de estudantes de Odontologia no Brasil ${ }^{1,6,7,9,15}$.

Durante sua trajetória histórica, a Odontologia caracterizou-se como uma profissão tipicamente masculina. No entanto, atualmente, tem-se discutido que ela passa por um processo crescente de feminização ${ }^{16}$. No presente estudo, as estudantes do sexo feminino compreenderam $69,81 \%$ dos participantes, essa feminização na Odontologia, também ressaltada por outros autores, é um fenômeno observado em diversas profissões ${ }^{1,7,10,17-21}$. De modo geral, a predominância de mulheres na Odontologia pode ser observada desde o final dos anos 90, sendo que há cerca de 40 anos a profissão poderia ser considerada eminentemente masculina, uma vez que $90 \%$ dos profissionais eram homens ${ }^{22}$.

$\mathrm{O}$ aumento no número de mulheres também tem sido observado nas universidades brasileiras, nas quais elas representam a maior parte dos estudantes do nível superior do país ${ }^{16}$. O termo feminização no mercado de trabalho se refere, normalmente, a um franco crescimento da população feminina em algumas profissões que historicamente eram desempenhadas pelos homens, como por exemplo Medicina e Odontologia $^{18}$. A profissionalização feminina, iniciada no século XIX, aconteceu relacionada aos papéis femininos tradicionais, ou seja, a mulher vinculada ao cuidar, ao educar e ao servir, entendidos como dom ou vocação ${ }^{23}$.

Em um estudo do perfil dos acadêmicos concluintes do curso de Odontologia em 2014 no estado da Bahia, os autores corroboram com essa tendência nos cursos de Odontologia. Segundo os mesmos houve predomínio do gênero feminino entre os acadêmicos das cinco instituições de ensino participantes da pesquisa ${ }^{19}$.

Em relação à idade dos estudantes, o fato de serem jovens pode estar relacionado com a idade em que ingressaram no curso $(68,23 \%$ tinham de 17 a 20 anos), e poucos relataram trancamento de matricula por algum período (3,33\%). Quanto ao estado civil observou-se que a maioria era solteira, refletindo uma tendência da população brasileira em casar-se mais tarde, priorizando a construção de uma formação profissional e inserção no mercado de trabalho ${ }^{2}$.

Em relação à condição socioeconômica das famílias, a maioria ganha entre 3 e 5 salários mínimos $(41,66 \%)$, semelhante ao observado por outros autores ${ }^{1,9}$. Entretanto esse valor foi inferior ao encontrado em estudo onde os alunos pesquisados tinham uma melhor condição socioeconômica ${ }^{6}$. Também foi observado que os pais possuem formação superior e/ou nível médio e estão inseridos no mercado de trabalho. Esses dados podem relacionar-se com a baixa faixa etária encontrada entre os estudantes, uma vez que, uma boa condição socioeconômica dos pais, permite aos alunos dedicar-se aos estudos durante o ensino médio e se preparar para o vestibular de Odontologia, provavelmente, sem precisar dividir seu tempo entre trabalho e estudos.

Muitos estudantes $(94,50 \%)$ afirmaram ter realizado monitorias, projetos de extensão e/ou pesquisa durante a graduação e $70,45 \%$ possuíam bolsas custeadas por esses programas institucionais. $\mathrm{O}$ mesmo foi identificado em um estudo com ingressantes e formandos da Faculdade de Odontologia da Araraquara, onde $51,2 \%$ dos formandos receberam bolsas durante o curso ${ }^{24}$. Um ciclo dinâmico e interativo em que a pesquisa aprimora e produz novos conhecimentos, os quais são difundidos por meio do ensino e da extensão, faz com que esses três pilares balizadores da formação universitária 
tornem-se complementares e interdependentes, atuando de forma sistêmica. A qualidade e o sucesso dos profissionais formados pelas universidades dependem, em grande parte, do nível de interação e articulação entre esses três pilares do conhecimento ${ }^{25}$.

Segundo os participantes, a opção pela Odontologia como profissão foi determinada principalmente pela realização pessoal e profissional, concordando com outros autores ${ }^{1,26}$. Em seguida, foram relatados interesse em atuar na comunidade, segurança e tranquilidade no futuro e a influência de cirurgiões-dentistas parentes e amigos. A presença de cirurgiõesdentistas na família foi observada em $25 \%$ dos estudantes formandos. Entretanto, estudo em Pernambuco apontou como principal motivo pela escolha do curso de Odontologia a identificação com a profissão ${ }^{9}$.

Em 2015, observou-se na Universidade Federal de Santa Catarina que a principal motivação que levou os alunos a optarem pela odontologia foi a admiração pela profissão, as motivações seguintes foram a vocação, a autonomia que a profissão oferta, a influência de alguém da família, ao prestigio e por ser uma profissão compensadora ${ }^{27}$.

Quando questionados sobre qual a principal finalidade da Odontologia, os estudantes evidenciaram a prevenção e manutenção da saúde bucal, promover higiene e estética e prevenção e tratamento. Esses dados parecem estar de acordo com a proposta dos cursos de Odontologia da UEPB, que é a formação de egresso com perfil de um profissional generalista, de sensibilidade social e competência técnica capazes de prestar atenção integral humanizada ${ }^{13,14}$. Achados similares foram observados em concluintes de Odontologia da Universidade Federal do Rio Grande do $\mathrm{Sul}^{7}$.

Com relação à satisfação com o curso, a maior parte dos estudantes do último semestre de Odontologia da UEPB mostraram-se satisfeitos com a opção pela profissão, assim como relatado por outros estudos ${ }^{18}$, e avaliaram o curso como bom ou ótimo. A maioria também considerou o tempo de graduação de cinco anos como adequado para a formação do cirurgião-dentista.

Na UEPB, mais da metade dos estudantes $(68,33 \%)$ afirmaram pretender atuar tanto no serviço público quanto no privado depois de formados. Essa intenção de conciliar o trabalho no serviço público com o privado também foi encontrada por outros autores ${ }^{26}$. Destaca-se que na Universidade Federal da Paraíba, em 2010, esse percentual chegou a $88,4 \%{ }^{28}$.

A prática exclusiva no consultório privado não se estabelece mais como uma realidade para a maior parte dos formandos. Tal situação pode ter sido influenciada pelas importantes e necessárias mudanças ligadas às políticas de Educação e de Saúde no país ${ }^{7,29,30}$. Porém, um estudo realizado na Universidade Federal de Campina Grande, em 2015, mostrou que 137 universitários $(66,6 \%)$ pretendiam atuar como autônomos em consultórios particulares ${ }^{21}$, resultado também observado por outros autores na Universidade Federal de Santa Catarina ${ }^{27}$.

Historicamente, a formação em Odontologia no Brasil baseou-se em um modelo autossuficiente, de transmissão de conteúdos e práticas, sem interlocução com as necessidades da sociedade. Como consequência, os cirurgiõesdentistas eram formados com uma visão frágil para legitimar conflitos pessoais e situações sociais. A crise no mercado liberal e a inclusão deste profissional na Estratégia de Saúde da Família fez surgir um novo mercado de trabalho na realidade do Sistema Único de Saúde (SUS). Nesse sentido, as DCN para o curso de Odontologia, implantadas em 2002, vieram propor mudanças no perfil de formação deste profissional, visando uma ressignificação de sua 
atuação e o desenvolvimento e melhor resolubilidade do SUS $^{31}$.

O ensino da Odontologia, a partir das DCN, passou a contemplar as necessidades sociais do país, fundamentando-se em relações sociais mais humanas e preparando o profissional a ser formado para o trabalho junto ao SUS, ou seja, um profissional qualificado, orientado pelo ensino baseado em evidência, bem treinado e comprometido com a igualdade na saúde ${ }^{3,32}$.

Destaca-se, que os motivos que levaram os estudantes a optar, na sua maioria, trabalhar tanto no setor público quanto no privado, foram a renda segura, a experiência nos primeiros anos de profissão até montar um consultório ou formar clientela e maior experiência profissional, semelhante aos resultados encontrados em estudantes da Universidade Federal do Rio Grande do Sul em 2014 .

Os estudantes se identificaram como trabalhadores de uma Equipe de Saúde da Família, com jornada de 40 horas semanais. Essa percepção sobre o mercado de trabalho pode ter sido influenciada pelo Projeto Pedagógico do Curso de Odontologia, que oferta como cenário de aprendizagem os estágios supervisionados no SUS, em seus diversos níveis de atenção, com o objetivo de desenvolver competências e habilidades para o trabalho em equipe, procurando desenvolver as atividades clínicas individuais e as ações com grupos operativos nos equipamentos sociais adscritos às unidades de Saúde da Família, buscando a integralidade da atenção em saúde bucal ${ }^{2}$. Em estudo realizado pela Universidade Federal do Ceará, os autores verificaram que $56 \%$ dos egressos do curso de Odontologia da instituição se inseriu no setor público ${ }^{3}$.

Em relação à realização de pós-graduação após o término do curso, quase a totalidade dos discentes de Odontologia da UEPB responderam afirmativamente $(96,67 \%)$, o que está de acordo com outros achados da literatura ${ }^{21,24}$. As áreas de maior interesse para especialização foram Prótese/Implantodontia, seguida de Endodontia e Ortodontia, com Cirurgia e Dentística aparecendo em terceiro lugar, semelhantemente aos resultados encontrados por outros autores ${ }^{21}$. Alguns estudos, porém, apontam cursos de pósgraduação nas especialidades de Cirurgia Bucomaxilofacial, Ortodontia e Implantodontia como sendo os mais desejados ${ }^{27,32}$.

Por fim, é necessário destacar que os resultados apresentados sobre o perfil do aluno graduando em Odontologia da UEPB referem-se a um curto período de tempo (um ano), e um monitoramento contínuo desses alunos é necessário para configurar o perfil do graduado de Odontologia na instituição.

Diante do exposto, consideramos interessante e necessária a realização de novos estudos na área, visto que a literatura sobre o tema ainda é escassa, exigindo maiores conhecimentos e um maior aprofundamento nesse campo de trabalho, para melhor compreender as questões relacionadas ao perfil dos acadêmicos de Odontologia, suas motivações e expectativas profissionais.

\section{CONCLUSÃO}

Os resultados demonstram que o perfil dos formandos em Odontologia da UEPB em 2016 é de mulher, jovem, solteira, sem filhos e natural da Paraíba, satisfeita com a opção pela Odontologia e com o curso de graduação de Odontologia da UEPB, com perspectiva de aliar o serviço público ao privado na atuação profissional, além de realizar cursos de pósgraduação.

\section{ABSTRACT \\ Profile of Dental graduates at the State University of Paraiba}

The purpose of this study was to identify the sociodemographic profile of the Dental graduates 
at the Universidade Estadual da Paraíba (UEPB), in 2016, the reasons that led them to opt for Dentistry, satisfaction with the course, as well as their perspectives of work and postgraduation. The sample was constituted by 60 students, who filled a semistructured questionnaire divided into three blocks: sociodemographic profile of the students; degree course in Dentistry; professional and postgraduate perspectives. The results showed a predominance of female, young, single, childless, originally from Paraíba, with monthly family income of 3 to 5 minimum wages and without a presence of dentists in the family. Their parents have either finished high school or higher education and are currently working. More than 90\% were satisfied for choosing dentistry. The reasons that made them choose this profession were mainly the professional performance and personal satisfaction, followed by the interest of working in the community. Approximately $40 \%$ of the students reported disapproval and few reported dropping classes, consequently not attending the courses for a semester or more. Approximately $80 \%$ of the students evaluated the course as good or excellent and considered the graduation time of five years as appropriate. The students intend to work both in the public service and in private practice and do postgraduate studies. The findings made it possible to identify the profile of the Dental graduates at UEPB, in 2016. It is recommended the permanent accompaniment of the trainees as an essential monitoring instrument of the students and the curriculum.

Descriptors: Dentistry. Education, Higer. Dental Students

\section{REFERÊNCIAS}

1. Granja GL, Santos JTL, Mariz RC, Araki AT, Souza SV, Nunes JMFF, Fonseca FRA Perfil dos estudantes de graduação em Odontologia: motivações e expectativas da profissão. Rev ABENO. 2016; 16(4):107-13.

2. Machado FC, Souto DMA, Freitas CHSM, Forte FDS Odontologia como escolha: perfil de graduandos e perspectiva para o futuro profissional. Rev ABENO. 2010; 10(2): 27-34.
3. Pinheiro FM, Nóbrega-Therrien SM, Almaida MEL, Almeida MI. A formação do cirurgião dentista no Brasil: contribuições de estudos para prática da profissão. RGO. 2009; 57(1):99-106.

4. Junges R, Stello RS, Portella FF, Rösing CK, Samuel SMW Impact of the implantation of a new curriculum in the process of learning in a Faculty of Dentistry in Brazil. Braz Oral Res. 2011; 25(6):478-84.

5. Rabelo ML, Bisionato C, Araújo MC, Griboski C, Meneghel SM. Educação Superior Brasileira: Perfil dos concluintes e sua percepção sobre a formação. R Est Inv Psico y Educ. 2015; 14:73-6.

6. Latreille AC, Sobrinho SM, Warmling AMF, Ribeiro DM, Amante CJ. Perfil socioeconômico dos graduandos em odontologia da Universidade Federal de Santa Catarina. Rev ABENO. 2015; 15(1):86-96.

7. Bockmann FS, Motta BB, Camargo JM, Petry PC, Toassi RFC. Perfil dos formandos de odontologia da Universidade Federal do Rio Grande do Sul e expectativas quanto a profissão, 2010-2011. RGO. 2014; 62(3) :267-74.

8. Sousa JE, Maciel LKB, Zocratto KBF O papel do ensino de graduação em Odontologia e o motivo da escolha da profissão: uma visão dos alunos concluintes. RFO UPF. 2013; 18(3):277-83.

9. Marques MD, Souza CA, Pazos CTC, Amaral DS, Vieira EG, Campus WCS, Carvalho EJA. Expectativas dos estudantes de odontologia quanto ao futuro profissional. Rev ABENO. 2015; 15(3):60-8.

10. Freire MCM, Jordão LMR, Ferreira NP, Nunes MF, Queiroz MG, Leles C R. Motivation towards career choice of brazilian freshman students in a fifteen-year period. J Dent Educ. 2011; 75(1):115-21.

11. Barbosa KGN, Dias JN, Cavalcante GMS, 
Nóbrega LM, Granville-Garcia AF, D’ávila S. Formação e Perspectiva do Mercado de Trabalho Sob o Olhar de Alunos de Odontologia Pesq Bras Odontoped Clin Integr. 2013; 13(1):89-94.

12. Aguiar CM, Pessoa MAV, Câmara AC, Perrier RA, de Figueiredo JA. Factors Involved in the Choice of Dentistry as an Occupation by Pernambuco Dental Students in Brazil. J Dent Educ. 2009; 73(9):1401-7.

13. Projeto Pedagógico de Curso PPC: Odontologia (Bacharelado). Universidade Estadual da Paraíba. CCBS; Núcleo docente estruturante. Campina Grande: UEPB, 2016.

14. Projeto Pedagógico de Curso PPC: Odontologia (Bacharelado). Universidade Estadual da Paraíba. CCTS; Núcleo docente estruturante. Araruna: UEPB, 2016.

15. Seminoff TADV, Borges AH, Volpato LER, Moretti MF, Silva NF, Vieira EMM, Segundo SA. Estudo da ansiedade e da condição socioeconômica em uma subpopulação de acadêmicos do curso de graduação em odontologia. ROBRAC. 2015; 24(71):219-22.

16. Friedrich EG. A presença feminina no curso de odontologia da UFSC. Trabalho de conclusão de curso. UFSC 2015.

17. Toassi RFC, Souza JM, Rosing CK, Baumgarten A. Perfil sociodemográfico e perspectivas em relação à profissão do estudante de Odontologia da Universidade Federal do Rio Grande do Sul, Brasil. Rev Fac Odontol Porto Alegre 2011;52(1):25-32.

18. Costa SM, Durões SJ, Abreu MHNG. Feminização do curso de Odontologia da Universidade Federal de Montes Claros. Ciênc Saúde Coletiva 2010; 15(1):1865-73.

19. Araújo DB, Campos EJ, Martins GB, Lima MJP, Araújo MTB. Perfil dos concluintes acadêmicos do curso de Odontologia em 2014 no Estado da Bahia. Rev Ciênc Méd
Biol. 2015; 14(2):198-205.

20. Andrade DLS, Prado FO. Conhecimento e atitudes de acadêmicos de odontologia sobre câncer bucal. Arch Healtd Invest. 2016; 5(2):90-7.

21. Costa CHM, Chacon LD, Lima ABL, Medeiros RSP, Almeida MSC. Perfil, motivos de ingresso e de evasão dos graduandos de Odontologia. Odontol Clín Cient, 2015; 14(3):713-8.

22. Funk PP, Flores MMDZ, Garbin CA, Hartmann MSM, Mendonça JL. Perfil do profissional formado pela Faculdade de Odontologia da Universidade de Passo Fundo/RS: da formação à realidade profissional. RFO UPF. 2004; 9(2):105-9.

23. Matos IB, Toassi RFC, Oliveira MC. Profissões e ocupações de saúde e o processo de feminização: tendências e implicações. Athenea Digital. 2013; 13(2):239-44.

24. Loffredo LCM, Pinelli C, Garcia PPNS, Scaf G, Camparis CM. Características socioeconômica, cultural e familiar de estudantes de Odontologia. Rev Odontol UNESP. 2004; 33(4):174-82.

25. Pivetta HMF, Backes DS, Carpes A, Battistel ALH, Marchiori M. Ensino, pesquisa e extensão universitária: em busca de uma integração efetiva. Linhas Críticas. 2010; 16(31):377-90.

26. Souza JM, Souza MG, Toassi RFC. Democratização ao serviço de educação pública a partir do REUNI - o curso noturno de Odontologia da Universidade Federal do Rio Grande do Sul. Rev PPGE UNESC 2015; 4(1):1-21.

27. Cardoso MB. As motivações acadêmicas e as expectativas profissionais dos alunos de Odontologia da UFSC. [Trabalho de conclusão de curso]. Florianópolis, Universidade Federal de Santa Catarina, 2015. 
28. Cavalcanti YW, Cartaxo RO, Padilha WWN. Educação odontológica e Sistema de Saúde brasileiro: práticas e percepções de estudantes de graduação. Arq Odontol. 2010; 46(4):224-31.

29. Garbin Mattevi GS, Carcereri D, Caetano JC. Odontologia e saúde suplementar: marco regulatório, políticas de promoção da saúde e qualidade da atenção. Ciênc Saúde Coletiva. 2013; 18(2):441-52.

30. Almeida-Filho N. Ensino superior e os serviços de saúde no Brasil. The Lancet: Saúde no Brasil. 2011; 377(9781):1898-900.
31. Dantas LLFS. Mudanças curriculares na formação em Odontologia no Brasil: Realidade ou intenção? [Trabalho de conclusão de curso]. Fortaleza. Universidade Estadual do Ceará, 2013.

32. Santos BRM, Gonzales PS, Carrer FCA, Araújo, ME. Perfil e expectativa dos ingressantes da universidade da USP: Uma visão integrada com as diretrizes curriculares nacionais e o sistema único de saúde. Rev ABENO. 2015; 15(1):28-37.

Correspondência para:

Gêisa Aiane de Morais Sampaio

e-mail: geisa_aiane@hotmail.com

Rua João Alves de Oliveira, 189 - Centro

58400-117 Campina Grande/PB 